\title{
Research Article \\ Schauder Fixed Point Theorem in Spaces with Global Nonpositive Curvature
}

\section{Constantin P. Niculescu and Ionel Rovenţa}

Department of Mathematics, University of Craiova, 200585 Craiova, Romania

Correspondence should be addressed to Ionel Rovenţa, roventaionel@yahoo.com

Received 3 June 2009; Accepted 9 November 2009

Recommended by Anthony To Ming Lau

The Schauder fixed point theorem is extended to the context of metric spaces with global nonpositive curvature. Some applications are included.

Copyright (C) 2009 C. P. Niculescu and I. Rovenţa. This is an open access article distributed under the Creative Commons Attribution License, which permits unrestricted use, distribution, and reproduction in any medium, provided the original work is properly cited.

\section{Introduction}

The aim of our paper is to discuss the extension of the Schauder fixed point theorem to the framework of spaces with global nonpositive curvature (abbreviated, global NPC spaces). A formal definition of these spaces is as follows.

Definition 1.1. A global NPC space is a complete metric space $E=(E, d)$ for which the following inequality holds true: for each pair of points $x_{0}, x_{1} \in E$ there exists a point $y \in E$ such that for all points $z \in E$,

$$
d^{2}(z, y) \leq \frac{1}{2} d^{2}\left(z, x_{0}\right)+\frac{1}{2} d^{2}\left(z, x_{1}\right)-\frac{1}{4} d^{2}\left(x_{0}, x_{1}\right)
$$

These spaces are also known as the Cat 0 spaces. See [1]. In a global NPC space, each pair of points $x_{0}, x_{1} \in E$ can be connected by a geodesic (i.e., by a rectifiable curve $\gamma:[0,1] \rightarrow$ $E$ such that the length of $\left.\gamma\right|_{[s, t]}$ is $d(\gamma(s), \gamma(t))$ for all $\left.0 \leq s \leq t \leq 1\right)$. Moreover, this geodesic is unique. The point $y$ that appears in Definition 1.1 is the midpoint of $x_{0}$ and $x_{1}$ and has the property

$$
d\left(x_{0}, y\right)=d\left(y, x_{1}\right)=\frac{1}{2} d\left(x_{0}, x_{1}\right) .
$$


Every Hilbert space is a global NPC space. Its geodesics are the line segments. The upper half-plane $\mathbf{H}=\{z \in \mathbb{C}: \operatorname{Im} z>0\}$, endowed with the Poincaré metric,

$$
d s^{2}=\frac{d x^{2}+d y^{2}}{y^{2}}
$$

constitutes another example of a global NPC space. In this case the geodesics are the semicircles in $\mathbf{H}$ perpendicular to the real axis and the straight vertical lines ending on the real axis.

A Riemannian manifold $(M, g)$ is a global NPC space if and only if it is complete, simply connected, and of nonpositive sectional curvature. Besides manifolds, other important examples of global NPC spaces are the Bruhat-Tits buildings (in particular, the trees). See [1]. More information on global NPC spaces is available in [2,3]. See also our papers [4-7].

In what follows $E$ will denote a global NPC space.

Definition 1.2. A set $C \subset E$ is called convex if $\gamma([0,1]) \subset C$ for each geodesic $\gamma:[0,1] \rightarrow E$ joining $r(0), \gamma(1) \in C$.

A function $\varphi: C \rightarrow \mathbb{R}$ is called convex if the function $\varphi \circ \gamma:[0,1] \rightarrow \mathbb{R}$ is convex for each geodesic $\gamma:[0,1] \rightarrow C, \gamma(t)=\gamma_{t}$, that is,

$$
\varphi\left(\gamma_{t}\right) \leq(1-t) \varphi\left(\gamma_{0}\right)+t \varphi\left(\gamma_{1}\right)
$$

for all $t \in[0,1]$.

One can prove that the distance function $d$ is convex with respect to both variables, a fact which implies that every ball in a global NPC space is a convex set. See [8, Corollary 2.5].

All closed convex subsets of a global NPC space are in turn spaces of the same nature.

The aim of the present paper is to discuss the extension of the Schauder fixed point theorem to the framework of global NPC spaces. The main result is as follows.

Theorem 1.3. Suppose that $C$ is a closed convex subset of a global NPC space $E$ with the property that the closed convex hull of every finite subset of $E$ is compact. Then every continuous map $T: C \rightarrow C$ whose image $T(C)$ is relatively compact has a fixed point.

The convex hull is defined in the context of global NPC spaces via the formula

$$
\operatorname{co} F=\bigcup_{n=0}^{\infty} F_{n}
$$

where $F_{0}=F$, and for $n \in \mathbb{N}^{\star}$ the set $F_{n}$ consists of all points in the ambient global NPC space which lie on geodesics which starts and end in $F_{n-1}$. The convex hull of a finite subset is not necessarily closed, but we can mention two important cases when this happens. The first one is that of Hilbert spaces. In fact, in any locally convex Hausdorff space, if $K_{1}, \ldots, K_{n}$ are compact convex subsets, then the convex hull of their union is compact too. See the monograph of Day [9, page 103]. The second case is provided by the following result.

Lemma 1.4. In a locally compact global NPC space, the closed convex hull of each finite family of points has the fixed point property. 
Recall that a topological space $X$ has the fixed point property if every continuous map from $X$ to itself has a fixed point.

Proof. In a locally compact global NPC space $E$, each bounded and closed convex subset is compact. In particular, the closed convex hull of each finite family of points is compact.

On the other hand, each NPC space is an absolute retract. In fact, each space with an upper curvature bound is an absolute neighborhood retract and an absolute neighborhood retract that is contractible is an absolute retract. To see this, recall that all the balls are convex in $E$ and all intersections of balls are empty or contractible. See [10, page 12]. Thus $E$ has arbitrary fine coverings with the property that all the intersections of the sets from each covering are empty or contractible. This implies that $E$ is an absolute neighborhood retract. See [11, Theorem 1.1(b)].

In order to end the proof we have to show that every compact absolute retract $E$ has the fixed point property. In fact, $E$ can be embeded topologically into the Hilbert cube $B$. According to the classical Schauder fixed point theorem (see [12, page 25]) $B$ has the fixed point property, hence also each retract of $B$, in particular $E$.

An immediate consequence of Lemma 1.4 is the following generalization of the Brouwer fixed point theorem.

Theorem 1.5. In a global NPC space, every compact convex set $K$ has the fixed point property.

Theorem 1.5 proves to be instrumental in our extension of the Schauder fixed point theorem. Our argument combines this result with the existence of some nicely behaved projections onto the compact convex subsets of a global NPC space. The details are presented in the next section.

In [7], the statement of Theorem 1.5 was mentioned without any reference and used to extend two others important results in convex analysis: the Knaster-KuratowskiMazurkievicz lemma and Fan's minimax inequality. The elegant proof indicated above was suggested to us by A. Lytchak.

The present paper ends with an application of Theorem 1.3 to the graph theory, by indicating a sufficient condition under which an edge-preserving map leaves fixed some vertex or an edge. Our result was inspired by previous work due to Kirk [13].

After this paper has been completed, Horvath has kindly informed us on his recent manuscript [14] that contains related results in the slightly more general context of complete quasi-Busemann spaces.

\section{Proof of Theorem 1.3}

We start noticing the existence of an analogue of the orthogonal projection in the framework of global NPC spaces.

Proposition 2.1 (see [8, Proposition 2.6]). (i) For each closed convex set $C$ in a global NPC space $E=(E, d)$ there exists a unique map $P: E \rightarrow C$ (projection onto $C$ ) such that

$$
d(P(x), x)=\inf _{c \in C} d(x, c) \quad \forall x \in E ;
$$


(ii) $P$ is orthogonal in the sense that

$$
d^{2}(x, c) \geq d^{2}(x, P(x))+d^{2}(P(x), c)
$$

for every $x \in E, c \in C$;

(iii) $P$ is nonexpansive,

$$
d(P(x), P(y)) \leq d(x, y) \text { for every } x, y \in E
$$

Based on Proposition 2.1 we can now prove that the compact subsets of a global NPC space are surprisingly special: they are "almost" finite-dimensional.

Corollary 2.2. Let $K$ be a compact subset of a global NPC space E. Then for each $\varepsilon>0$ there exists a finite subset $F$ of $K$ and a function $P: K \rightarrow \overline{\mathrm{CO}} F$ such that $d(P(x), x)<\varepsilon$ for all $x \in K$.

Proof. Since $K$ is compact, for $\varepsilon>0$ arbitrarily fixed there is a finite subset $F=\left\{x_{1}, x_{2}, \ldots, x_{m}\right\}$ $\subset K$ such that

$$
K \subset \bigcup_{1 \leq i \leq m} B_{\varepsilon}\left(x_{i}\right)
$$

Let $P$ be the orthogonal projection of $E$ onto the closed convex set $C=\overline{\mathrm{co}} F$. According to Proposition 2.1(ii), for each $x \in K$ we have

$$
d(P(x), x) \leq d(x, C)<\varepsilon
$$

and the proof is done.

We are now in a position to prove Theorem 1.3.

For this, let us denote by $K$ the closure of $T(C)$. By our hypotheses, $K$ is a compact set and thus Corollary 2.2 applies. Hence for each natural number $n$ we may choose a finite subset $F_{n}$ of $K$ and a map $P_{n}: K \rightarrow \overline{\mathrm{co}} F_{n}$ such that

$$
d\left(P_{n}(x), x\right)<\frac{1}{n} \quad \forall x \in K
$$

Since $\overline{\mathrm{co}} F_{n} \subset C$, we may consider the maps

$$
T_{n}: \overline{\mathrm{co}} F_{n} \longrightarrow \overline{\mathrm{co}} F_{n}, \quad T_{n}(x)=P_{n}(T(x))
$$

By Theorem 1.5, each of these maps $T_{n}$ has a fixed point, say $z_{n}$. Since $K$ is compact, we may assume (by passing to a subsequence if necessary) that the sequence $\left(T\left(z_{n}\right)\right)_{n}$ is convergent to a point $z \in C$.

We will prove that $z$ is a fixed point for the function $T$. In fact, by applying (2.6) for $x=T\left(z_{n}\right)$, we get $d\left(T_{n}\left(z_{n}\right), T\left(z_{n}\right)\right)<1 / n$, and so the sequences $\left(z_{n}\right)_{n}$ and $\left(T\left(z_{n}\right)\right)_{n}$ both have 
the limit $z$. Since $T$ is continuous we conclude that $T(z)=\lim _{n \rightarrow \infty} T\left(z_{n}\right)=z$. This ends the proof of Theorem 1.3.

As a consequence we can state the following analogue of the Leray-Schauder principle.

Theorem 2.3. Let E be a global NPC space with the property that the closed convex hull of every finite subset is compact and let $f: E \rightarrow E$ be a map that verifies the following two conditions:

(i) $f$ maps the bounded sets into relatively compact sets;

(ii) there exists a closed ball $\bar{B}_{r}\left(x_{0}\right)$ such that every point $x \in S_{r}\left(x_{0}\right)$ with $f(x) \notin \bar{B}_{r}\left(x_{0}\right)$ is outside the geodesic segment of endpoints $x_{0}$ and $f(x)$.

Then $f$ has a fixed point.

Proof. Let $P_{C}: E \rightarrow E$ be the orthogonal projection onto $C=\bar{B}_{r}\left(x_{0}\right)$. According to our hypothesis (i), the image of the map $f^{*}=\left.P_{C} \circ f\right|_{C}: C \rightarrow C$ is relatively compact, and by Theorem 1.3 there must be a point $x^{*}$ such that $f^{*}\left(x^{*}\right)=x^{*}$. If $f\left(x^{*}\right) \in C$, then $x^{*}=f^{*}\left(x^{*}\right)=f\left(x^{*}\right)$ and thus $f$ has a fixed point.

Suppose that $f\left(x^{*}\right) \notin C$. Because $P_{C}\left(f\left(x^{*}\right)\right)=x^{*}$ we infer that $d\left(x_{0}, x^{*}\right)=$ $d\left(x_{0}, P_{C}\left(f\left(x^{*}\right)\right)\right)=r$, and $x^{*}=P_{C}\left(f\left(x^{*}\right)\right)$ is a point on the geodesic joining the points $x_{0}$ and $f\left(x^{*}\right)$. This contradicts (ii). The proof is done.

The classical Leray-Schauder principle refers to the maps $f$ from a Banach space $E$ into itself that verifies the condition (i) above and the following substitute of the condition (ii): There exists a number $r>0$ such that $\|x\|=r$ implies $f(x) \neq \lambda x$ for all $\lambda>1$.

Applications of the classical Leray-Schauder principle to partial differential equations can be found in many books. See, for example, [12].

Theorem 2.3 easily yields the following criterion for the existence of fixed points.

Corollary 2.4. Let E be a global NPC space with the property that the closed convex hull of every finite subset is compact and let $f: \bar{B}_{r}\left(x_{0}\right) \rightarrow$ E be a map that verifies the following two conditions:

(i) $f$ maps the bounded sets into relatively compact sets;

(ii) $f\left(S_{r}\left(x_{0}\right)\right) \subset \bar{B}_{r}\left(x_{0}\right)$.

Then $f$ has a fixed point.

In recent years much activity was done to build NPC-analogues of classical function spaces. However, few things are known in connection to partial differential equations. See $[15,16]$.

Instead, we will mention here that exactly the same argument we used for Theorem 1.3 yields also the classical fixed point theorem of Browder, Gohde, and Kirk concerning the nonexpansive maps.

Theorem 2.5. Let $C$ be a closed, bounded, convex set in a real Hilbert space. Then each nonexpansive map $T: C \rightarrow C$ has at least one fixed point.

See $[17,18]$, for other interesting results concerning the existence of fixed points in uniformly convex metric spaces. 


\section{An Application to $\mathbb{R}$-Trees}

A graph is an ordered pair $(V, E)$, where $V$ is a set and $E$ is a binary relation on $V(E \subset V \times V)$. The elements of $V$ are called vertices, and the elements of $E$ are called edges.

Definition 3.1. Let $(V, E)$ be a graph. A map $f: V \rightarrow V$ is said to be edge-preserving if $(a, b) \in E$ implies $(f(a), f(b)) \in E$.

The map $f: E \rightarrow E$ leaves an edge fixed if there exists an edge $(a, b) \in E$ which coincides with the edge $(f(a), f(b)) \in E$.

Definition 3.2 (J. Tits). An $\mathbb{R}$-tree is a metric space $(T, d)$ such that

(i) there is a unique geodesic segment $[x, y]$ for each pair of points $x, y \in T$;

(ii) if $[y, x] \cap[x, z]=\{x\}$, then $[y, x] \cup[x, z]=[y, z]$.

A complete $\mathbb{R}$-tree is a global NPC space with the property that the closed convex hull of every finite subset is compact. As a consequence we can specialize the Leray-Schauder principle (Theorem 2.3 above) to the context of complete $\mathbb{R}$-trees.

Lemma 3.3. Let $(T, d)$ be a complete $\mathbb{R}$-tree, and let $f: T \rightarrow T$ be a map that verifies the following two conditions:

(i) f maps bounded sets into relatively compact sets;

(ii) there exists a closed convex subset $K$ of $T$ and a vertex $p_{0} \in \operatorname{int} K$ such that $x \notin\left[p_{0}, f(x)\right]$, for every $x \in \partial K$.

Then $f$ has a fixed point.

An application is given in the next theorem.

Theorem 3.4. Let $V=(V, E)$ be a connected reflexive graph with no cycle. We attach to the graph $V$ an $\mathbb{R}$-tree $T=(T, d)$ by identifying each edge by a unit interval of the real line and assigning the shortest path distance to any pair of points of $T$. With respect to this metric, $T$ is complete. Let $f: T \rightarrow T$ be an edge-preserving map which verifies the conditions (i) and (ii) of Lemma 3.3. Then $f$ leaves fixed either an vertex of $V$ or an edge of $E$.

Proof. By definition, $p \in \partial T$ if $p$ is a boundary point of $V$. According to Lemma 3.3, $f$ admits a fixed point $z$. Two possibilities may occur: either $z$ is a vertex of $V$, or $z$ lies properly on the unit interval joining the vertices of some edge $[x, y] \in E$. If $f$ fails to leave some vertex of $V$ fixed, then necessarily $z$ is the midpoint of the metric interval $[x, y]$ with $f(x)=y$ and $f(y)=x$. But in this case $[x, y]$ is a fixed edge of $f$.

\section{An Open Question}

In what follows we discuss an example built on the global NPC space $\operatorname{Sym}^{++}(n, \mathbb{R})$ (of all $n \times n$ dimensional positive definite matrices with real coefficients), when endowed with the trace metric, 
Fixed Point Theory and Applications

$$
d_{\text {trace }}(A, B)=\left(\sum_{k=1}^{n} \log ^{2} \lambda_{k}\right)^{1 / 2}
$$

here $\lambda_{1}, \ldots, \lambda_{n}$ are the eigenvalues of $A B^{-1}$. Since similarities preserve eigenvalues, this metric is invariant under similarities, that is,

$$
d_{\text {trace }}(A, B)=d_{\text {trace }}\left(C^{-1} A C, C^{-1} B C\right) \quad \forall C \in \operatorname{GL}(n, \mathbb{R})
$$

Notice that $A B^{-1}$ is similar with

$$
A^{-1 / 2}\left(A B^{-1}\right) A^{1 / 2}=A^{1 / 2} B^{-1 / 2}\left(A^{1 / 2} B^{-1 / 2}\right)^{\star}>0
$$

and this fact assures the positivity of the eigenvalues of $A B^{-1}$.

A proof that $\operatorname{Sym}^{++}(n, \mathbb{R})$ is a Cartan-Hadamard manifold (and thus a global NPC space) may be found in [19]. See also [20].

Given two matrices $A$ and $B$ in $\operatorname{Sym}^{++}(n, \mathbb{R})$, we may consider the map

$$
T(X)=B X^{-1} A,
$$

whose unique fixed point is the geometric mean of $A$ and $B$, that is,

$$
A \sharp B=A^{1 / 2}\left(A^{-1 / 2} B A^{-1 / 2}\right)^{1 / 2} A^{1 / 2} \text {. }
$$

Moreover $A \sharp B$ is the unique matrix $C$ in $\operatorname{Sym}^{++}(n, \mathbb{R})$ such that

$$
d_{\text {trace }}(A, C)=d_{\text {trace }}(B, C)=\frac{1}{2} d_{\text {trace }}(A, B)
$$

The existence of $A \sharp B$ seems to escape both to Theorems 1.3 and 2.5 above. We leave open the question of proving a stronger fixed point result that encompasses the existence of $A \sharp B$ and the two aforementioned theorems.

\section{Acknowledgments}

The authors gratefully acknowledge helpful correspondence on topological convexities and fixed points from Alexander Lytchak and Charles Horvath. This work has been supported by the National University Research Council through the research project 420/2008.

\section{References}

[1] M. R. Bridson and A. Haefliger, Metric Spaces of Non-Positive Curvature, vol. 319 of Grundlehren der Mathematischen Wissenschaften, Springer, Berlin, Germany, 1999. 
[2] W. Ballmann, Lectures on Spaces of Nonpositive Curvature, DMV Seminar Band 25, Birkhäuser, Basel, Switzerland, 2005.

[3] J. Jost, Nonpositive Curvature: Geometric and Analytic Aspects, Lectures in Mathematics ETH Zürich, Birkhäuser, Basel, Switzerland, 1997.

[4] C. P. Niculescu, "An extension of the Mazur-Ulam theorem," in Proceedings of the International Workshop on Global Analysis and Applied Mathematics, K. Tas, D. Krupka, O. Krupkova, and D. Baleanu, Eds., vol. 729 of AIP Conference Proceedings, pp. 248-256, American Institute of Physics, Ankara, Turkey, April 2004.

[5] C. P. Niculescu, "The Krein-Milman theorem in global NPC spaces," Bulletin Mathématique de la Société des Sciences Mathématiques de Roumanie. Nouvelle Série, vol. 50(98), no. 4, pp. 343-346, 2007.

[6] C. P. Niculescu, "The Hermite-Hadamard inequality for convex functions on global NPC space," Journal of Mathematical Analysis and Applications, vol. 356, no. 1, pp. 295-301, 2009.

[7] C. P. Niculescu and I. Rovenţa, "Fan's inequality in geodesic spaces," Applied Mathematics Letters, vol. 22, no. 10, pp. 1529-1533, 2009.

[8] K. T. Sturm, "Probability measures on metric spaces of nonpositive curvature," in Heat Kernels and Analysis on Manifolds, Graphs, and Metric Spaces: Lecture Notes from a Quarter Program on Heat Kernels, Random Walks, and Analysis on Manifolds and Graphs, P. Auscher, T. Coulhon, and A. Grigor'yan, Eds., vol. 338 of Contemporary Mathematics, pp. 357-390, Paris, France, 2003.

[9] M. M. Day, Normed Linear Spaces, Ergebnisse Der Mathematik Und Ihrer Grenzgebiete, Springer, New York, NY, USA, 3rd edition, 1973.

[10] A. Lytchak and V. Schroeder, "Affine functions on CAT(k)-spaces," Mathematische Zeitschrift, vol. 255, no. 2, pp. 231-244, 2007.

[11] H. Torunczyk, "Concerning locally homotopy negligible sets and characterization of $l_{2}$-manifolds," Fundamenta Mathematicae, vol. 101, no. 2, pp. 93-110, 1978.

[12] R. F. Brown, A Topological Introduction to Nonlinear Analysis, Birkhäuser, Boston, Mass, USA, 1993.

[13] W. A. Kirk, "Fixed point theorems in CAT(0) spaces and R-trees," Fixed Point Theory and Applications, vol. 2004, no. 4, pp. 309-316, 2004.

[14] Ch. Horvath, "A note on metric spaces with continuous midpoints," Annals of the Academy of Romanian Scientists, Series on Mathematics and Its Applications, vol. 1, no. 2, 2009.

[15] L. Ambrosio, N. Gigli, and G. Savaré, Gradient Flows in Metric Spaces and in the Space of Probability Measures, Lectures in Mathematics ETH Zürich, Birkhäuser, Basel, Switzerland, 2nd edition, 2008.

[16] J. Heinonen, Lectures on Analysis on Metric Spaces, Universitext, Springer, New York, NY, USA, 2001.

[17] R. Espinola, A. Fernandez Leon, and B. Piatek, "Fixed points of single and set valued mappings in uniformly convex metric spaces with no metric convexity," Fixed Point Theory and Applications, vol. 2010, Article ID 169837, 16 pages, 2010.

[18] Z. D. Mitrović, "On almost coincidence points in generalized convex spaces," Fixed Point Theory and Applications, vol. 2006, Article ID 91397, 7 pages, 2006.

[19] S. Lang, Fundamentals of Differential Geometry, vol. 191 of Graduate Texts in Mathematics, Springer, New York, NY, USA, 1999.

[20] C. P. Niculescu and L.-E. Persson, Convex Functions and Their Applications: A Contemporary Approach, vol. 23 of CMS Books in Mathematics, Springer, New York, NY, USA, 2006. 